\title{
Eta and eta' physics in KLOE
}

\author{
Marcin Berlowski ${ }^{1,2}$ on behalf of KLOE-2 Collaboration \\ ${ }^{1}$ Istituto Nazionale di Fisica Nucleare, Frascati, Italy \\ ${ }^{2}$ National Centre for Nuclear Research, Warsaw, Poland
}

\begin{abstract}
We present results obtained by the KLOE Collaboration at the DAФNE $\mathrm{e}^{+} \mathrm{e}^{-}$collider located in Frascati Laboratories, Italy. The results concern precise measurements of several $\eta$ meson decays such as Dalitz slope parameters and charge asymmetries for $\eta \rightarrow \pi^{+} \pi^{-} \pi^{\circ}$, branching ratio and plane asymmetry for $\eta \rightarrow \pi^{+} \pi^{-} \mathrm{e}^{+} \mathrm{e}^{-}$, and first observation of $\eta \rightarrow \mathrm{e}^{+} \mathrm{e}^{-} \mathrm{e}^{+} \mathrm{e}^{-}$along with its branching ratio determination. Also preliminary studies of $\eta \rightarrow \pi^{\circ} \gamma \gamma$ decay channel are presented as well as physics perspectives for many different processes for the ongoing KLOE-2 data taking.
\end{abstract}

\section{Introduction}

The KLOE experiment operates at DAФNE, the Frascati $\phi$-factory. DAФNE is an $\mathrm{e}^{+} \mathrm{e}^{-}$ collider running at the center of mass energy of the $\phi$ meson $(1020 \mathrm{MeV})$ that is produced nearly at rest. The first data campaign between 2001 and 2006 collected $\sim 2.5 \mathrm{fb}^{-1}$ on peak data and $\sim 0.26 \mathrm{fb}^{-1}$ at $20 \mathrm{MeV}$ below the mass of the $\phi$ meson. The data taking period started on November 2014 [1], after several accelerator and detector upgrades [2], aims to collect more than $5 \mathrm{fb}^{-1}$ for the end of March 2018 .

The detector consists of a large cylindrical Drift Chamber, surrounded by a leadscintillating fiber Electromagnetic Calorimeter embedded both in magnetic field of $\sim 0.52 \mathrm{~T}$. The $4 \mathrm{~m}$ in diameter and $3.3 \mathrm{~m}$ long drift chamber filled with a mixture of $90 \%$ Helium and $10 \%$ Isobutane provides the position with resolutions of $\sigma_{\mathrm{xy}} \sim 150 \mu \mathrm{m}$ and $\sigma_{\mathrm{z}} \sim 2 \mathrm{~mm}$. The momentum resolution $\frac{\sigma_{p t}}{p t}$ is below the $0.4 \%$ for the charged tracks with a scattering angle between $45^{\circ}$ and $135^{\circ}$ [3]. The calorimeter is divided into a barrel and two endcaps covering $98 \%$ of the solid angle providing energy and time resolution $\frac{\sigma_{E}}{E}=\frac{5.7 \%}{\sqrt{E(\mathrm{GeV})}}$ and $\sigma_{T}=\frac{57 \mathrm{ps}}{\sqrt{E(\mathrm{GeV})}} \oplus 140 \mathrm{ps}$, respectively [4].

\section{$2 \eta \rightarrow \pi^{+} \pi^{-} \pi^{\circ}$}

The isospin violating $\eta \rightarrow \pi^{+} \pi^{-} \pi^{\circ}$ decay can be used as a test bench in several topics such as: Chiral Perturbation Theory (ChPT), for a low-energy QCD effective field theory, and as a precise probe of the $\mathrm{u}-\mathrm{d}$ quark mass difference. The decay amplitude is proportional to light quark mass ratio (in $\mathrm{ChPT} \Gamma \sim \mathrm{Q}^{-4}$ ): 


$$
Q^{2} \equiv \frac{m_{s}^{2}-\widehat{m}^{2}}{m_{d}^{2}-m_{u}^{2}}, \text { where } \widehat{m}=\frac{1}{2}\left(m_{d}+m_{u}\right)
$$

The Eq. 1 is valid up to NLO ChPT. After correcting for the electromagnetic effects at the lowest order by using a combination of kaon and pion masses, a $Q$ value $=24.2$ is obtained. The theoretical calculations show a very slow convergence of the ChPT effective field theory decay widths [5]: $\Gamma_{\mathrm{LO}}=66 \mathrm{eV}$ and $\Gamma_{\mathrm{NLO}}=160-210 \mathrm{eV}$. Those values can be compared to the experimental results $\Gamma_{\text {exp }}=300 \pm 12 \mathrm{eV}$ showing discrepancy that could originate from higher order contributions to the decay amplitude or from corrections to the $\mathrm{Q}$ value. In order to provide input for the dispersive calculations and to clarify the discrepancy between theory [6-8] and the experiments [9-14], more high statistic measurements are required.

The Dalitz plot density in the $\eta$ meson rest frame can be parametrized using Taylor expansion around $\mathrm{X}=\mathrm{Y}=0$ :

$$
|A(X, Y)|^{2} \approx 1+\mathrm{a} Y+\mathrm{b} Y^{2}+\mathrm{c} X+\mathrm{d} X^{2}+\mathrm{e} X Y+\mathrm{f} Y^{3}+\mathrm{g} X^{2} Y+\cdots
$$

where $X=\sqrt{3} \frac{T_{\pi+}-T_{\pi-}}{Q}, Y=\frac{3 T_{\pi^{\circ}}}{Q}-1, Q=T_{\pi+}+T_{\pi-}+T_{\pi^{\circ}} . T_{\pi^{\circ \pm}}$ are the kinetic energies of the final state $\pi^{\circ \pm}$ particles. Moreover C-invariance excludes terms with odd powers of $X$. The parameters $a, b, \ldots$ in the Eq. 2 are going to be extracted by fitting the experimental Dalitz plot distribution.
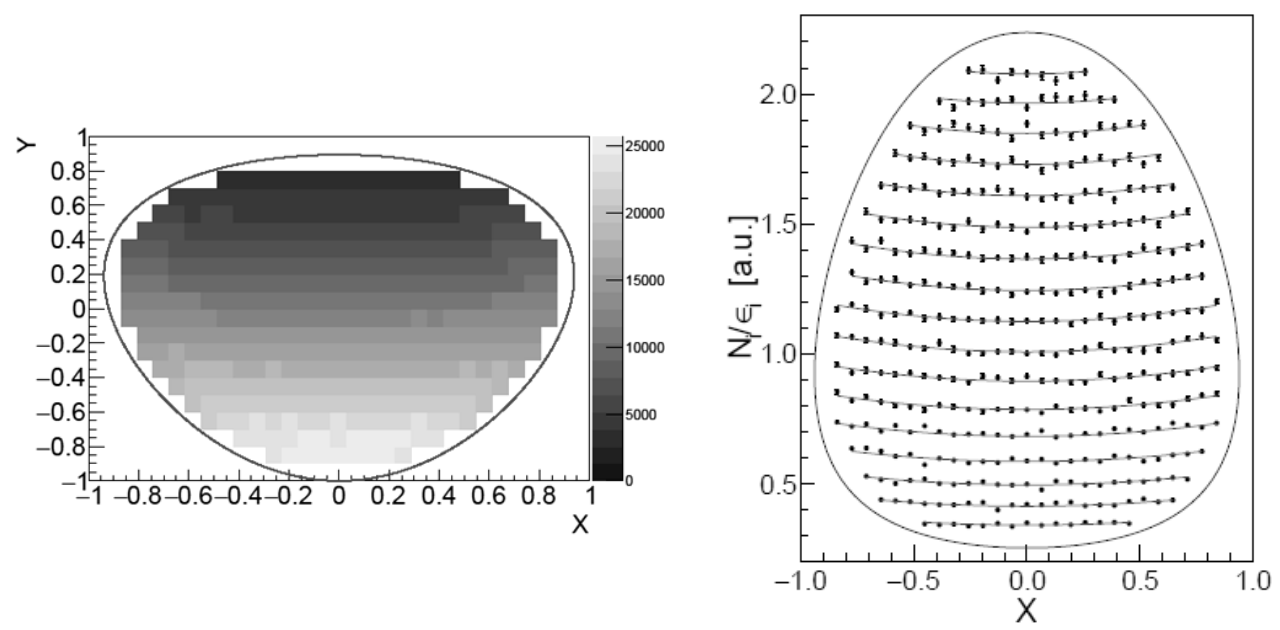

Fig. 1. Left: background-subtracted Dalitz plot of data. Right: acceptance corrected Dalitz plot data together with the result of the fit. Only statistical uncertainties are shown.

The analysis was based on $1.7 \mathrm{fb}^{-1}$ data collected. The events containing two oppositely charged tracks and three neutral clusters originating from the interaction point were selected. The highest-energy photon coming from the 2-body $\phi$ decay $\phi \rightarrow \eta \gamma$, were selected by requiring $\mathrm{E}_{\gamma}>250 \mathrm{MeV}$. Discrimination against electron contamination from Bhabha scattering were achieved by the time-of-flight measurement of the clusters connected to the charged tracks in the electromagnetic calorimeter. Additional cuts have been applied in order to reduce the remaining background: a) on the angle between $\pi^{+}$or $\pi^{-}$and the closest photon from $\pi^{\circ}$ decay, $b$ ) on the angle between clusters in the $\pi^{\circ}$ rest frame, and c) on the reconstructed $\pi^{\circ}$ invariant mass squared. Signal selection efficiency of $37.6 \%$ was obtained with signal to background ratio $S / N=133$ in the final data sample with less than $1 \%$ of residual background. The Dalitz plot distribution was constructed using 31 and 20 bins for $X$ and $Y$ respectively and consisted of $\sim 4.7 \cdot 10^{6}$ events in total. The bin width of the Dalitz plot was determined both by the resolution in $X$ and $Y$ and the number of events in each of 
the bins yielding to the 371 bins in the final plot. Left panel of the Fig.1 shows data distribution after background subtraction and the acceptance corrected data are presented on the right panel of Fig. 1.

The final results for the Dalitz plot parameters, including influence of systematic effects, are:

$$
\begin{gathered}
a=-1.095 \pm 0.003_{-0.002}^{+0.003} \\
b=+0.145 \pm 0.003 \pm 0.005 \\
d=+0.081 \pm 0.003_{-0.005}^{+0.006} \\
f=+0.141 \pm 0.007_{-0.008}^{+0.007} \\
g=-0.044 \pm 0.009_{-0.013}^{+0.012}
\end{gathered}
$$

This results are in agreement with the previous KLOE results [11] improving the accuracy up to a factor 3. On top of that this is the first statistically significant measurement of the $Y^{2} X$ term (g parameter). In addition tests of $\mathrm{C}$ conservation in the decay were performed by studying asymmetries for the unbinned data. All the results are consistent with zero. For example the asymmetry between kinetic energies of positively and negatively charged pions, $\mathrm{A}_{\mathrm{LR}}$, was found to be:

$$
A_{L R}=\left(-5.0 \pm 4.5_{-11}^{+5.0}\right) \times 10^{-4}
$$

The new KLOE-2 data, at the moment the most precise available, are being used as input for the dispersive analyses, for example in determination of the quark mass ratio $\mathrm{Q}=$ $22.0 \pm 0.7$ by the Bern group [15].

\section{$3 \eta \rightarrow e^{+} e^{-} e^{+} e^{-}$}

The $\eta \rightarrow \mathrm{e}^{+} \mathrm{e}^{-} \mathrm{e}^{+} \mathrm{e}^{-}$decay has been observed for the first time [16]. The theoretical values for the branching ratio (BR) for the decay are based on the triangle anomaly $[17,18]$, which predicts them to be in the range $2.4-2.6 \times 10^{-5}$. The left panel of Fig. 2 shows that the decay is also important to constrain the transition form factor of the $\eta$ meson [19]. The measurement is based on an integrated luminosity of $1.7 \mathrm{fb}^{-1} \phi$ decays analyzing the chain $\phi \rightarrow \eta\left(\rightarrow \mathrm{e}^{+} \mathrm{e}^{-} \mathrm{e}^{+} \mathrm{e}^{-}\right) \gamma$, with the monochromatic photon of energy $\mathrm{E}_{\gamma} \sim 363 \mathrm{MeV}$. The selection required exactly one high energy photon with $\mathrm{E}>250 \mathrm{MeV}$ and at least four charged tracks coming from the point of the beams collision. Monte Carlo signal simulations were made according to Bijnens and Persson [19] and included Final State Radiation calculations. In order to reduce the background coming from the photon conversion for the decays such as $\eta \rightarrow \gamma \mathrm{e}^{+} \mathrm{e}^{-}$or $\eta \rightarrow \gamma \gamma$ the rejection method based on the invariant mass and the distance between $\mathrm{e}^{+} \mathrm{e}^{-}$tracks at the beam pipe or at the drift chamber walls was implemented. Processes with a $\pi^{+} \pi^{-}$pair misidentified as $\mathrm{e}^{+} \mathrm{e}^{-}$(e.g. $\left.\phi \rightarrow \pi^{+} \pi^{-} \pi^{\circ}\left(\rightarrow \gamma \mathrm{e}^{+} \mathrm{e}^{-}\right)\right)$are reduced by measuring the time of flight of the charged particles with the electromagnetic calorimeter. The remaining residual background is mainly due to radiative Bhabha events with photon conversion: $\mathrm{e}^{+} \mathrm{e}^{-} \rightarrow \mathrm{e}^{+} \mathrm{e}^{-} \gamma \gamma\left(\rightarrow \mathrm{e}^{+} \mathrm{e}^{-}\right)$.

The right panel of Fig. 2 shows the two components fit of the data spectrum after selection. Background and signal are parametrized by a third order polynomial function and by two Gauss functions respectively.

The fit result yields to $362 \pm 29$ signal events, with $\chi^{2} / \mathrm{ndf}=43.9 / 34$, with a confidence level of $12 \%$. Taking into account the signal efficiency of $20 \%$ together with systematic effects we obtain:

$$
\operatorname{BR}\left(\eta \rightarrow \mathrm{e}^{+} \mathrm{e}^{-} \mathrm{e}^{+} \mathrm{e}^{-}(\gamma)\right)=\left(2.4 \pm 0.2_{\text {stat }} \pm 0.1_{\text {syst }}\right) \times 10^{-5}
$$


where the main systematic errors are due to photon conversion effects and the stability of results with different fit binning.
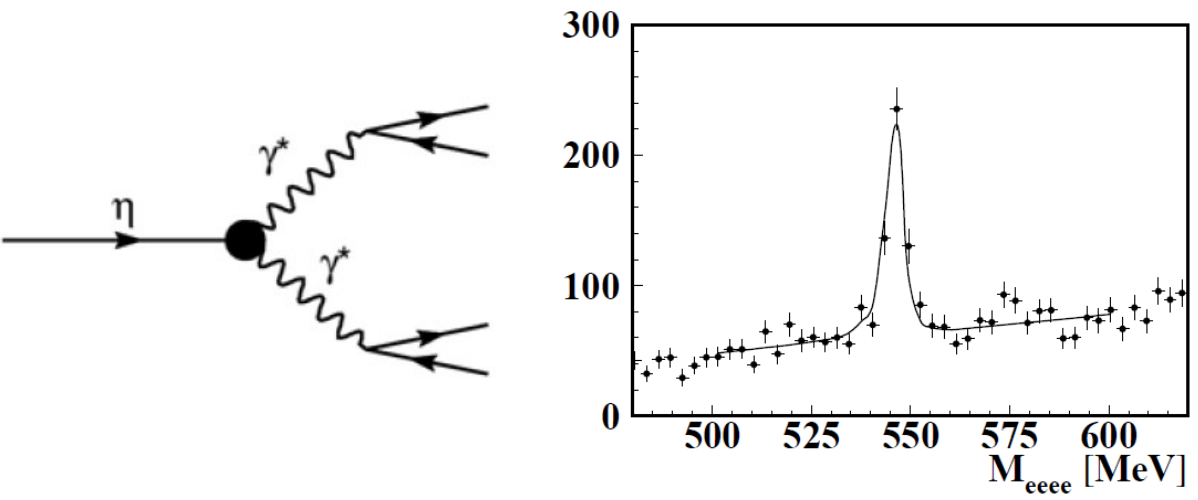

Fig. 2. Left: Feynman diagram for the $\eta \rightarrow \mathrm{e}^{+} \mathrm{e}^{-} \mathrm{e}^{+} \mathrm{e}^{-}$decay. Right: fit to the $\mathrm{e}^{+} \mathrm{e}^{-} \mathrm{e}^{+} \mathrm{e}^{-}$invariant mass.

\section{$4 \eta \rightarrow \pi^{+} \pi^{-} \mathrm{e}^{+} \mathrm{e}^{-}$}

The $\eta \rightarrow \pi^{+} \pi^{-} \mathrm{e}^{+} \mathrm{e}^{-}$decay allows to probe the electromagnetic structure of the $\eta$ meson and to compare the predictions of the branching ratio values based on different theoretical models such as Vector Meson Dominance, Chiral Perturbation Theory or chiral effective Lagrangian [17, 20-22]. It also allows to search for a possible CP violation beyond the Standard Model predictions by measuring the angular asymmetry between pions and electrons decay planes [23]. The analysis has been performed on $1.7 \mathrm{fb}^{-1}$ data collected on the $\phi$ meson peak and $0.26 \mathrm{fb}^{-1}$ off-peak data used for continuous background estimation. Effects of Final State Radiation have been taken into account using dedicated simulation package at the event generation level in order to fully account for radiative decays. Moreover the signal MC has been generated according to the matrix element described in [23] assuming null $A_{\phi}$ value. Only events containing two pairs of oppositely charged pairs and one neutral calorimeter cluster of energy greater than $250 \mathrm{MeV}$ were selected. In order to distinguish between pions and electron time-of-flight measurement using the time information from the calorimeter was used. To improve the energy and momentum resolution even further the kinematic fit is performed imposing the four-momentum conservation and the time-of-flight of the monochromatic photon of $363 \mathrm{MeV}$. Background contribution was evaluated performing a fit on the sidebands of the $\pi^{+} \pi^{-} \mathrm{e}^{+} \mathrm{e}^{-}$invariant mass with background shapes. To reject the events due to photon conversion the $\mathrm{e}^{+} \mathrm{e}^{-}$pairs were extrapolated down to the intersection with the beam pipe and rejected based on their invariant mass and their distance from the interaction point. For the signal estimate the $\eta$ mass region between 535 and $555 \mathrm{MeV}$ was considered (the left panel of Fig. 3), performing the event counting after background subtraction. The resulting number of signal events, $\mathrm{N}_{\pi \pi \mathrm{e}}=1555 \pm 52$, was used to extract the branching ratio [24]:

$$
\operatorname{BR}\left(\eta \rightarrow \pi^{+} \pi^{-} \mathrm{e}^{+} \mathrm{e}^{-}(\gamma)\right)=\left(26.8 \pm 0.9_{\text {stat }} \pm 0.7_{\text {syst }}\right) \times 10^{-5}
$$

The decay plane asymmetry $\mathrm{A}_{\phi}$ was defined as the sign asymmetry of the quantity $\sin \phi \cdot \cos \phi$, were $\phi$ here is the angle between the pion and the electron planes in the $\eta$ meson rest frame. It has been evaluated for the events in the signal region after background subtraction. The value obtained is:

$$
\mathrm{A}_{\phi}=\left(-0.6 \pm 2.5_{\text {stat }} \pm 1.8_{\text {syst }}\right) \times 10^{-2}
$$


This is the first measurement of this asymmetry. The distribution of the $\sin \phi \cdot \cos \phi$ variable is shown in the right panel of Fig. 3.
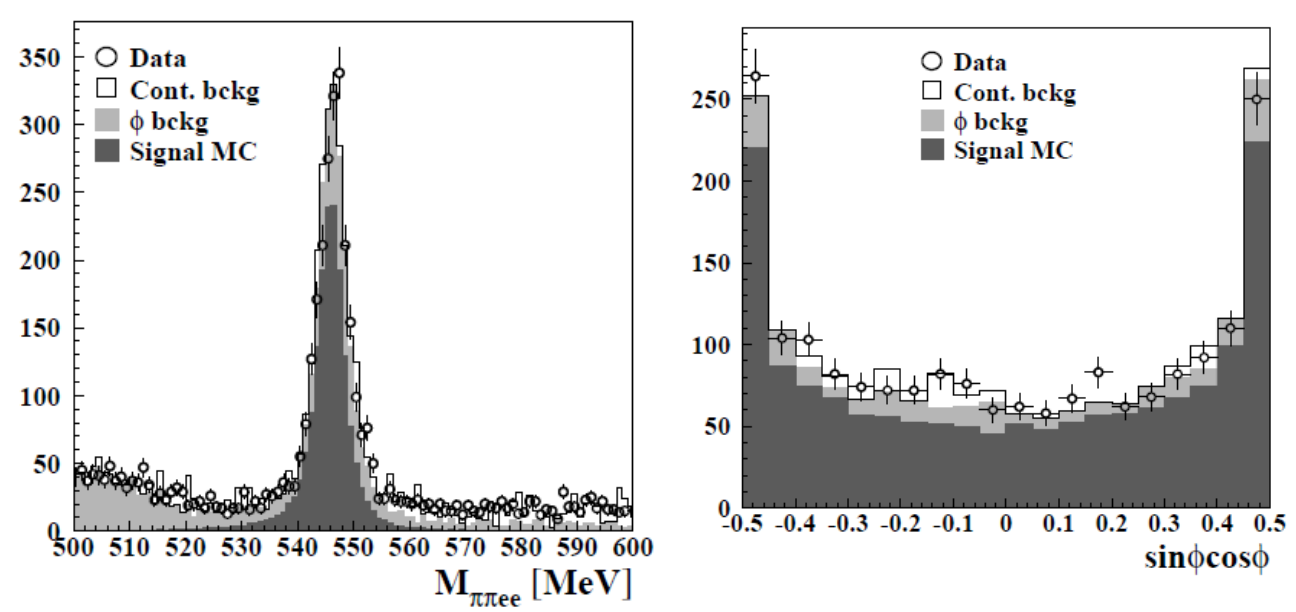

Fig. 3. Right: $\pi^{+} \pi^{-} \mathrm{e}^{+} \mathrm{e}^{-}$invariant mass spectrum zoomed around the $\eta$ meson mass. Left: distribution of the $\sin \phi \cdot \cos \phi$ variable in the signal region. For both histograms dots represent data, the black histogram is the expected distribution, i.e. signal simulation (dark grey), $\phi$ background (light grey) and continuum background (white).

\section{$5 \eta \rightarrow \pi^{\circ} \gamma \gamma$}

The $\eta \rightarrow \pi^{\circ} \gamma \gamma$ decay is very interesting both from the experimental and theoretical point of view. The theory made impressive progress since last 40 years when the first very large values for the branching ratio were obtained [25]. The experimental value of this branching fraction [26-28] has been decreasing with time, along with the increase of machine luminosities and the available statistics of the $\eta$ meson samples produced, showing that the main issue in the measurement of the branching fraction is the correct background estimate.

At KLOE this decay proceeds via $\phi \rightarrow \eta \gamma$, therefore we are looking for the final state with 5 prompt (aligned in time) photons and no charged tracks in the same time window. The analysis was based on $450 \mathrm{pb}^{-1}$ data sample collected during 2001-2002. The main background processes are radiative decays of the $\phi$ meson with either $\mathrm{a}_{0}$ or $\mathrm{f}_{0}$ in the final state, direct production of $\omega$ meson $\mathrm{e}^{+} \mathrm{e}^{-} \rightarrow \omega\left(\rightarrow \pi^{\circ} \gamma\right) \pi^{\circ}$ and $\eta$ meson decay into $3 \pi^{\circ}$, when some of the photons are lost or merged into another clusters. The composition of the background in the data sample is determined at an early stage of the analysis when the signal contribution is negligible, by fitting the spectrum of the invariant mass of all photon pairs $\left(\mathrm{m}_{\gamma \gamma}\right)$. Further selection criteria include constrained kinematic

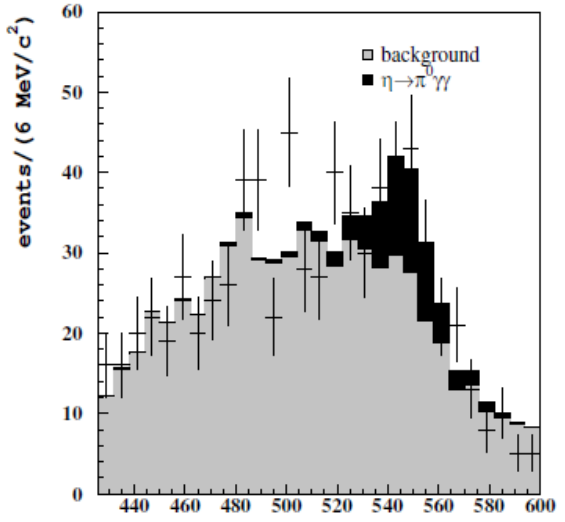

Fig. 4. The $\mathrm{m}_{4 \gamma}$ distribution: the dots with error bars show the data; simulated signal and backgrounds are normalized according to the fit results. fits to reject the background and likelihood function to identify merged clusters. As a last 
step of the analysis the spectrum of $\mathrm{m}_{4 \gamma}$ is used to extract the number of signal events $\mathrm{N}_{\text {sig }}=$ $68 \pm 23$. The spectrum, shown in Fig. 4, is fitted with the Monte Carlo expected distributions for signal and backgrounds. Using MC evaluated efficiency of $(4.63 \pm 0.09) \%$ as well as $\eta \rightarrow 3 \pi^{\circ}$ as normalization channel we obtain the preliminary result for the $\eta \rightarrow \pi^{\circ} \gamma \gamma$ branching ratio [29]:

$$
\operatorname{BR}\left(\eta \rightarrow \pi^{\circ} \gamma \gamma\right)=\left(8.4 \pm 2.7_{\text {stat }} \pm 1.4_{\text {syst }}\right) \times 10^{-5}
$$

\section{Conclusions and outlook}

KLOE-2 data taking at DA $\phi N E$ is in progress, since November 2014 and $4 \mathrm{fb}^{-1}$ of data has been already integrated. By the end of the experiment data taking in March 2018, an acquired luminosity of $5 \mathrm{pb}^{-1}$ will be easily collected. This will allow us to continue with increased statistics the precision study of the light meson properties such as precise measurement of the Dalitz plot parameters of $\eta \rightarrow \pi^{+} \pi^{-} \pi^{\circ}$ or Branching Ratios of rare $\eta$ meson decays exploiting a sample of $\sim 2.5 \cdot 10^{8}$ eta's collected.

\section{References}

1. G. Amelino-Camelia et al., Eur. Phys. J. C 68, 619-681 (2010)

2. M Zobov et al., Phys. Rev. Lett. 104, 174801 (2010)

3. M. Adinolfi et al., Nucl. Instrum. Meth. A 461, 25-28 (2001)

4. F. Ambrosino et al., Nucl. Instrum. Meth. A 598, 239-243 (2009)

5. K. Kampf, M. Knecht, J. Novotný, M. Zdráhal, Phys. Rev. D 84, 114015 (2011)

6. G. Colangelo, S. Lanz, H. Leutwyler, E. Passemar, PoS EPS-HEP2011 304 (2011)

7. P. Guo et al., Phys. Rev. D 92, 054016 (2015)

8. M. Gormley et al., Phys.Rev. D 2, 501-505 (1970)

9. J. Layter et al., Phys.Rev. D 7, 2565-2568 (1973)

10. CRYSTAL BARREL collaboration, A. Abele et al., Phys. Lett. B 417, 197-201 (1998)

11. KLOE collaboration, F. Ambrosino et al., JHEP 0805, 006 (2008)

12. WASA-AT-COSY collaboration, P. Adlarson et al., Phys. Rev. C 90, 045207 (2014)

13. BESIII collaboration, M. Ablikim et al., Phys. Rev. D 92, 012014 (2015)

14. G. Colangelo, S. Lanz, H. Leutwyler, E. Passemar, Phys. Rev. Lett. 118, 022001 (2017)

15. KLOE-2 Collaboration, A. Anastasi et al., JHEP 1605, 019 (2016)

16. KLOE \& KLOE-2 Collaborations, F. Ambrosino et al., Phys. Lett. B 702, 324 (2011)

17. C. Jarlskog, H. Pilkuhn, Nucl. Phys. B 1, 264-268 (1967)

18. L. G. Landsberg, Phys. Rept. 128, 301 (1985)

19. J. Bijnens, F. Persson, hep-ph/0106130

20. A. Faessler, C. Fuchs, M. I. Krivoruchenko, Phys. Rev. C 61, 035206 (2000)

21. C. Picciotto, S. Richardson, Phys. Rev. D 48, 3395 (1993)

22. B. Borasoy, R. Nissler, Eur. Phys. J. A 33, 95 (2007)

23. D. N. Gao, Mod. Phys. Lett. A 17, 1583 (2002)

24. KLOE \& KLOE-2 Collaborations, F. Ambrosino et al., Phys. Lett. B 675, 283 (2009)

25. L. Ametller, J. Bijnens, A. Bramon, F. Cornet, Phys. Lett. B 276, 185 (1992)

26. D. Alde et al., Z. Phys. C 25, 225 (1984)

27. S. Prakhov et al., Phys. Rev. C 72, 025201 (2005)

28. N. Knecht et al., Phys. Lett. B 589, 14 (2004)

29. KLOE collaboration, B. Di Micco et al., Acta Phys. Slov. 56, 403 (2006) 